\title{
Acute fibrinous and organizing pneumonia with myelodysplastic syndrome and pneumocystis jiroveci pneumonia: a case report
}

\author{
Minjie Lin ${ }^{1,2}$, Yingwei Zhang ${ }^{3}$, Yuying Qiu ${ }^{1}$ \\ ${ }^{1}$ Department of Pulmonary and Critical Care Medicine, Nanjing Drum Tower Hospital, Clinical College of Nanjing Medical University, Nanjing, \\ China; ${ }^{2}$ Department of Critical Care Medicine, The Second Hospital of Nanjing, Nanjing University of Chinese Medicine, Nanjing, China; \\ ${ }^{3}$ Department of Pulmonary and Critical Care Medicine, Nanjing Drum Tower Hospital, The Affiliated Hospital of Nanjing University Medical \\ School, China \\ Correspondence to: Yuying Qiu. Department of Pulmonary and Critical Care Medicine, Nanjing Drum Tower Hospital, Clinical College of Nanjing \\ Medical University, Nanjing 210008, China. Email: njqiuyuying@163.com.
}

\begin{abstract}
Acute fibrinous and organizing pneumonia (AFOP) is an unusual pathological pattern which is characterized by intra-alveolar deposition of fibrin (fibrin ball) and organizing pneumonia in a scattered distribution, and the pathological diagnosis plays an irreplaceable role in the diagnosis. Most Patients cannot confirm etiology, till now, known etiology included connective tissue disease, infection, environmental and occupational exposure, drugs, organ transplant, and tumor. It can be divided into acute and subacute subtype according to the extent of progress. The most common symptoms of AFOP were fever, cough, and dyspnea. Bilateral consolidations and ground-glass opacities (GGO) usually can be seen on chest CT images. At present, the treatment protocol for AFOP has not reached a consensus Glucocorticoid, immunosuppressants, stem cell transplantation or lung transplantation may contribute to improved clinical outcome. Here, we report a case of AFOP with myelodysplastic syndrome and pneumocystis jiroveci pneumonia (PJP). After treatments of glucocorticoid, immunosuppressant, chemotherapy, antibiotics and blood transfusion, the patient's clinical symptoms, peripheral blood test, and imaging findings were obviously improved. In this case, we consider the AFOP was caused by MDS and the immunodeficiency after chemotherapy lead to secondary PJP. This typical case highlights the importance of appropriate therapy for coexisted diseases of those patients with refractory AFOP.
\end{abstract}

Keywords: Acute fibrinous and organizing pneumonia (AFOP); myelodysplastic syndrome; pneumocystis jiroveci pneumonia (PJP); case report

Submitted Nov 25, 2020. Accepted for publication Feb 24, 2021.

doi: 10.21037/apm-20-2344

View this article at: http://dx.doi.org/10.21037/apm-20-2344

\section{Introduction}

Acute fibrinous and organizing pneumonia (AFOP) is an unusual histological pattern, which was first described by Beasley (1). Previously, AFOP was considered as a form of diffuse alveolar damage (DAD), eosinophilic pneumonia (EP) or bronchiolitis obliterans with organizing pneumonia (BOOP). Nowadays, it is recognized as a distinct pattern of lung injury in pathology, which is characterized by intraalveolar deposition of fibrin (fibrin ball) and organizing pneumonia in a scattered distribution (2). The prognosis of
AFOP was poor.

Myelodysplastic syndrome is a heterogeneous group of clonal stem cell disorders, featured by useless hematopoiesis in different cell lineages, and has the potential to translate into acute myeloid leukemia (AML). Patients with MDS are usually dormant, and the diagnosis is made by bone marrow aspiration showing cytopenia or dysplasia. Treatment options include chemotherapy and bone marrow transplantation (3). MDS often leads to lung infection because of leukopenia, but MDS with AFOP is rare. Among some cases of hematopathy with AFOP, MDS is a risk factor, 

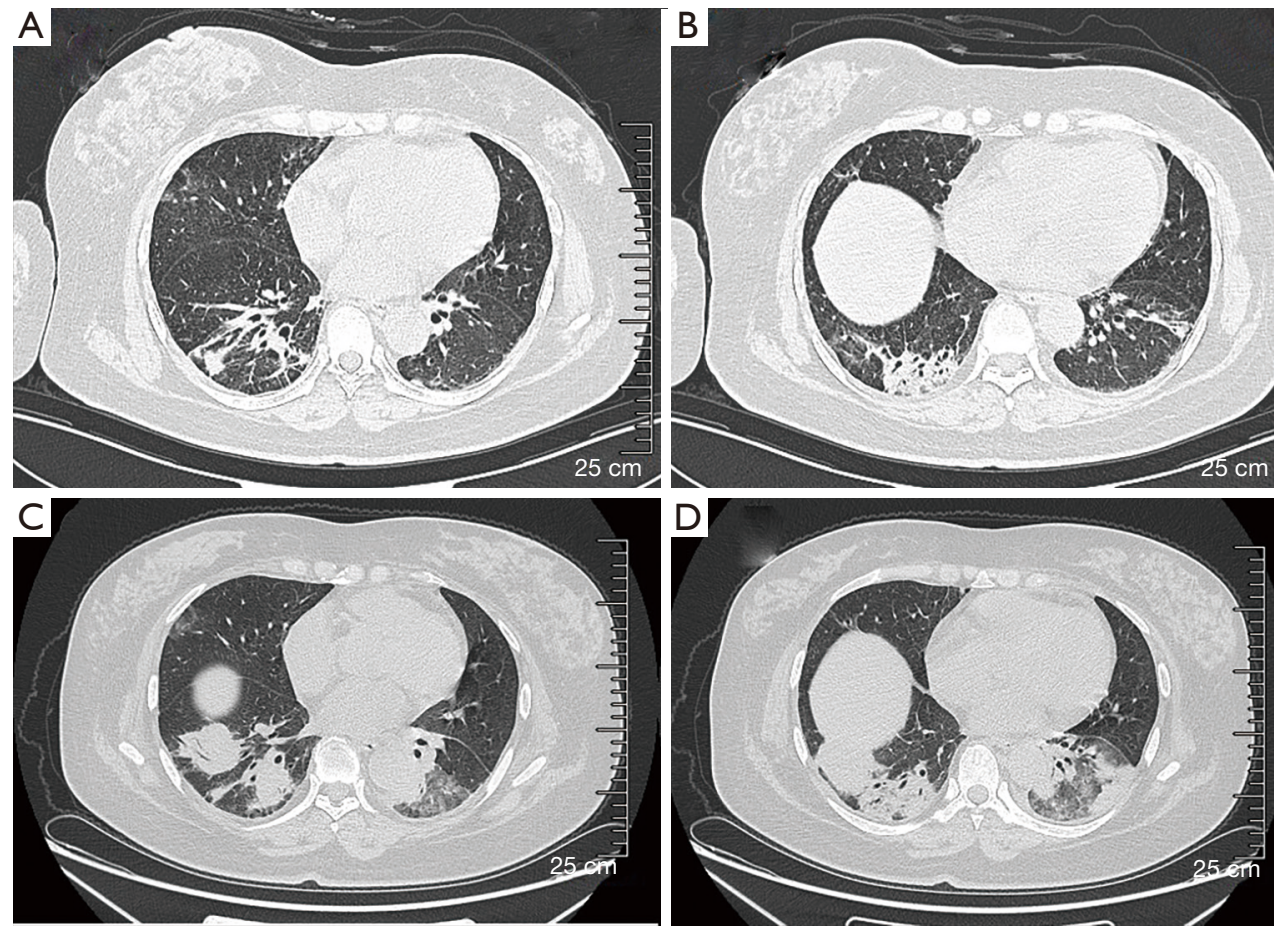

Figure 1 The chest CT scan during the department of pulmonary and critical care medicine. (A,B) Chest CT scan showed that bilateral consolidations and ground-glass opacities (GGO) at the bottom of the lungs; (C,D) chest CT scan showed that bilateral consolidations and GGO at the bottom of the lungs are greater than last time.

possibly due to autoimmunity. Here, we present a case of AFOP with myelodysplastic syndrome, which translated into AML and secondary pneumocystis jiroveci infection in the course of the disease. We present the following article in accordance with the CARE reporting checklist (available at http://dx.doi.org/10.21037/apm-20-2344).

\section{Case presentation}

A 49-year-old woman was hospitalized in November 2017 with complains of intermittent fever, cough, and dyspnea for two months. Before admission, her chest computed tomography (CT) scans revealed bilateral consolidations and ground-glass opacities (GGO) at the bottom of the lungs. Antibiotics were administered in a local hospital for several days but her symptoms persisted. Then transbronchial lung biopsy (TBLB) was done, histological examination supported the diagnosis of AFOP. Then $80 \mathrm{mg}$ methylprednisolone per day was administered and the patient responded very well. However, once the dosage was reduced to $40 \mathrm{mg} /$ day, the same symptoms reoccurred. The patient was also diagnosed with MDS via bone marrow aspirate in the local hospital because of low hemoglobin level. She has no history of poison exposure, smoking or alcohol intake. After admission to hospital, her vital signs were recorded: body temperature $36.7^{\circ} \mathrm{C}$, blood pressure $174 / 113 \mathrm{mmHg}$, heart rate 105 beats/min, respiratory rate 19 breaths/min. Pulmonary auscultation revealed mild crackles in the right lower lung fields and other physical examinations were unremarkable. Laboratory tests showed white blood count (WBC) was $6.5 \times 10^{9} / \mathrm{L}$ (neutrophil $81.5 \%$ ), hemoglobin $(\mathrm{Hb})$ was $76 \mathrm{~g} / \mathrm{L}$, platelet (Plt) count was $200 \times 10^{9} / \mathrm{L}$, C-reactive protein (CRP) was $111.6 \mathrm{mg} / \mathrm{L}$, erythrocyte sedimentation rate (ESR) was $150 \mathrm{~mm} / \mathrm{h}$. Procalcitonin (PCT), Brain natriuretic peptide (BNP), hepatic function, renal function, autoantibody, and anti-neutrophil cytoplasmic antibodies were all normal. Pulmonary function test showed mildly restrictive pulmonary dysfunction and moderately decreased diffusion capacity. Doppler echocardiography revealed enlarged left atria, slight mitral and tricuspid valve regurgitation, and mild pulmonary hypertension. Chest CT scan showed bilateral consolidations and GGO at the bottom of the lungs (Figure $1 A, B)$. Arterial blood gas analysis without oxygen 

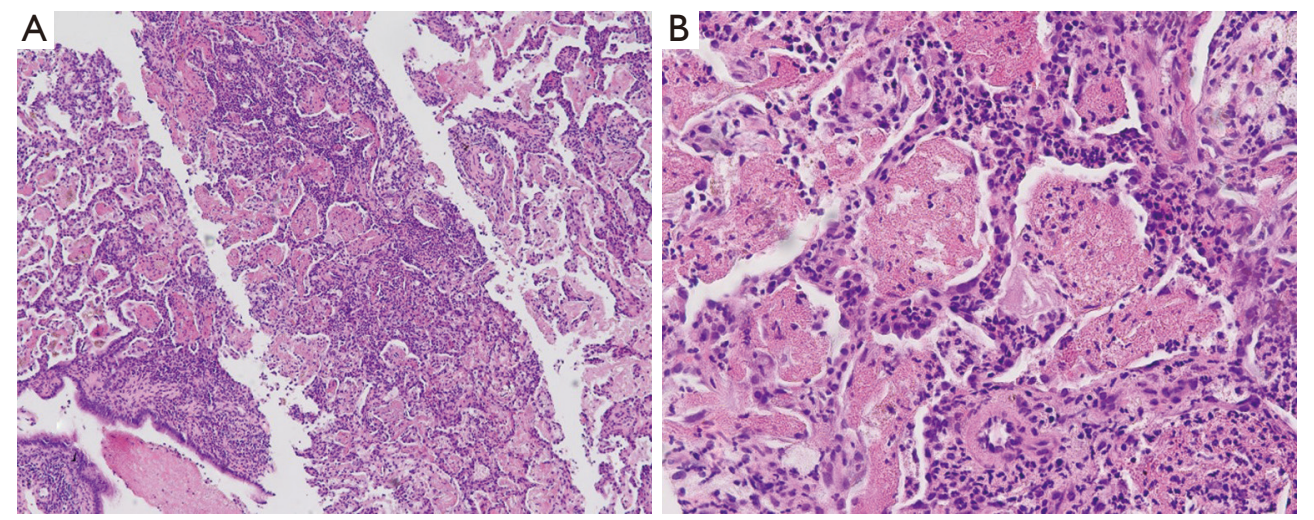

Figure 2 Pathological findings of percutaneous needle biopsy on December 18, 2018. (A) A large amount of neutrophil infiltration, hyaline membrane or eosinophil infiltration was observed (HE staining, $\times 10$ ); (B) the interalveolar space was slightly widened while a large amount of fibrinoid deposits in the alveoli (HE staining, $\times 40)$.

therapy demonstrated $\mathrm{PaCO}_{2}$ of $39.9 \mathrm{mmHg}, \mathrm{PaO}_{2}$ of $66 \mathrm{mmHg}$ and $\mathrm{PH}$ of 7.474 . Considering clinical symptoms, pulmonary image, and pathological features, the diagnosis of AFOP was established. methylprednisolone $40 \mathrm{mg}$ per day and clarithromycin $500 \mathrm{mg}$ per day were initiated and the patient's symptoms were improved rapidly after treatment. Then the dosage of glucocorticoids was reduced to Prednisone $35 \mathrm{mg} /$ day and the patient was discharged. Unfortunately, she was readmitted after a month because of symptom recurrence. Laboratory investigations showed $\mathrm{WBC}$ was $16.2 \times 10^{9} / \mathrm{L}$ (neutrophil $73.0 \%$ ), Hb was $67 \mathrm{~g} / \mathrm{L}$, Plt was $200 \times 10^{9} / \mathrm{L}$, ESR was $140 \mathrm{~mm} / \mathrm{h}$, CRP was $166 \mathrm{mg} / \mathrm{L}$, The PCT, EpsteinBarr Virus-DNA, T-SPOT, BNP, 1,3- $\beta$-D glucan test, Galactose mannitol glycan antigen test was normal. Chest CT scan showed that bilateral consolidations and GGO progressed compared with last time (Figure 1C,D). To identify the new lesions on CT images, percutaneous needle biopsy (PCNB) was performed on December 18, 2018, and tissue pathology (left lung) showed large amount of neutrophil infiltration, the interalveolar space was slightly widened and a large amount of fibrinoid deposits in the alveoli, which was consistent with AFOP (Figure 2A,B). After diagnosis, $500 \mathrm{mg}$ methylprednisolone per day was administered for 3 days followed by $60 \mathrm{mg}$ for 6 days then $40 \mathrm{mg}$ for 5 days, cyclosporine A and cyclophosphamide were used at the same time. Antibiotics included Piperacillin and Tazobactam, Moxifloxacin and Ganciclovir were also used to cover common Gramnegative bacilli and cytomegalovirus, the patient's symptoms were improved after treatment. Repeated routine blood tests showed that $\mathrm{WBC}$ was $40.3 \times 10^{9} / \mathrm{L}, \mathrm{Hb}$ was $58 \mathrm{~g} / \mathrm{L}$, Plt was $50 \times 10^{9} / \mathrm{L}$, peripheral blood smear revealed $42 \%$ blast cells. The department of hematology considered the progression of MDS to AML. So, the patient was referred to the department of hematology on January 07, 2019 for future examinations. Bone marrow cytology showed $78 \%$ blast cells. The chromosome was 46.XX. NO P53 or 5q deletion were detected, 43 common fusion genes of AML test showed Hox 11 gene was positive. Therefore, the patient was diagnosed with AML-M2a. CAG chemotherapy was initiated on January 10, 2019, but fever comes back after treatment, Blood cell count was reexamined showing that WBC was $1.8 \times 10^{9} / \mathrm{L}$, lymphocyte was $0.8 \times 10^{9} / \mathrm{L}$. A chest CT scan showed a new onset bilateral extensive GGO (Figure 3A,B), which was consistent with PJP. Cotrimoxazole was incorporated into the treatment plan, then her temperature returned to normal gradually and the chest CT scan showed the lesions of GGO with consolidation was absorbed before her discharge (Figure 3C,D). She received regular chemotherapy in a local hospital. At an 8-month follow-up, her pulmonary and hematological condition were stable without complains of any symptoms and the dosage of oral methylprednisolone was reduced to $12 \mathrm{mg}$ per day. She was appreciating our treatments. All procedures performed in this study involving human participants were in accordance with the ethical standards of the institutional and national research committees, and with the Helsinki Declaration (as revised in 2013). Informed consent was obtained from the patient for this publication. 

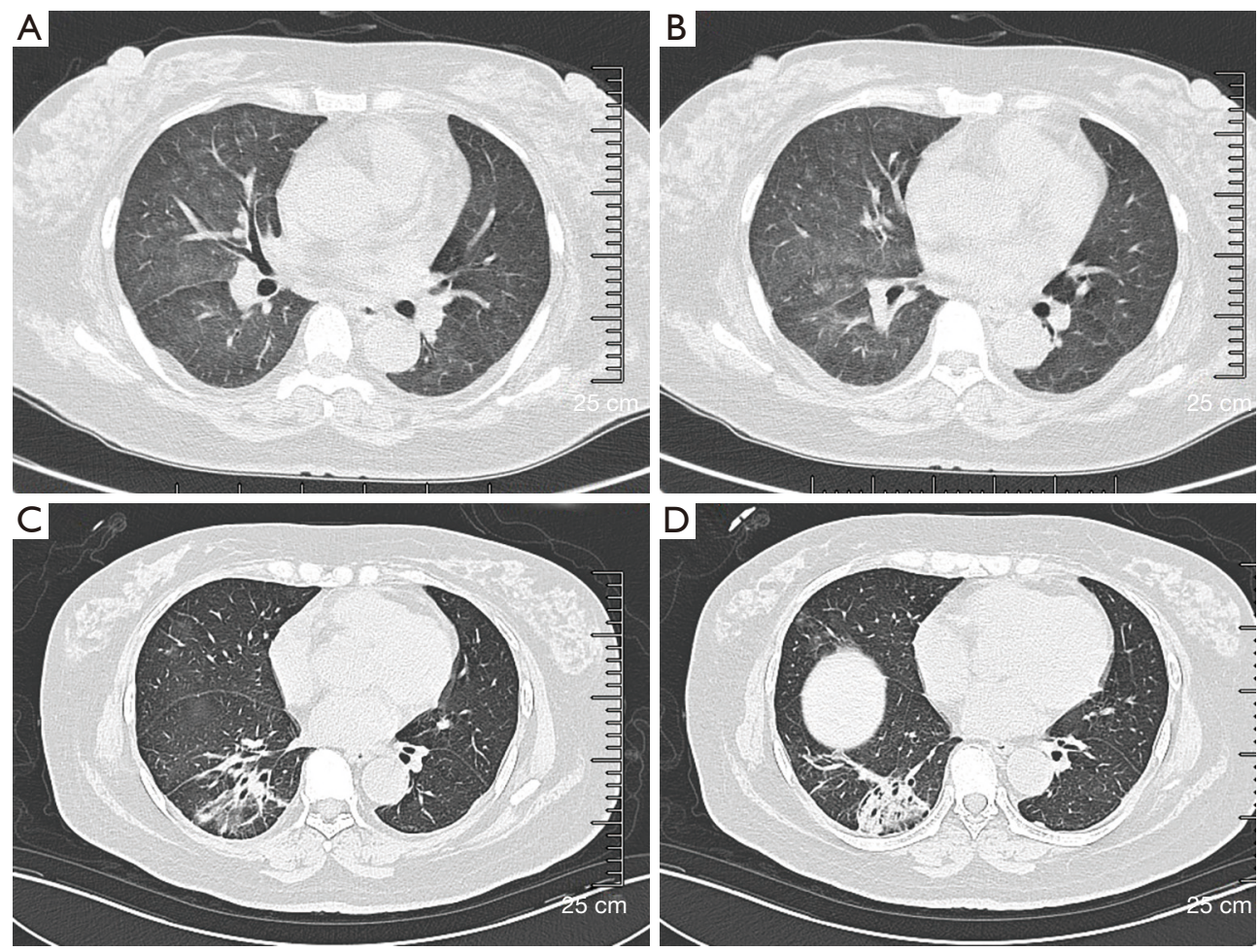

Figure 3 Chest CT scan during the department of hematology. (A,B) Chest CT scan showed that new extensive ground-glass opacities (GGO) appeared around both lungs; (C,D) chest CT scan showed that the lesion of GGO with consolidation was absorbed.

\section{Discussion}

AFOP was a rare histological entity, most literature were case reports. As far as we know, this is the first report of AFOP in a patient with MDS and PJP, and Corticosteroid, immunosuppressor, antibiotics, and chemotherapy led to its successful control.

After reviewing 111 patients of AFOP, Kuza et al. (4) concluded that most cases cannot confirm etiology, known causes included connective tissue disease, infection, environmental and occupational exposure, drugs, organ transplant, and tumor. As for this case, we considered AFOP was caused by MDS. The patient had same symptoms repeatedly when the dosage of glucocorticoids was tapered, but after treating AML from MDS transformation, patient's symptoms were controlled and no repeated symptoms occurred during the follow-up period. However, infection cannot be completely ruled out, because some special pathogens were insidious and cannot be covered by commonly used antibiotics. Therefore, long-term followup is necessary, especially if the AML could be cured in the future.
AFOP can be divided into acute and subacute according to the extent of progress. The acute mode usually progresses to respiratory failure rapidly and leads to death. Unlike acute mode, the progression of subacute mode was relatively moderate and most patients had better prognosis after treatment (1). Our patient was classified into the pattern of subacute, because the progression of her disease was relatively moderate and she didn't develop to respiratory failure.

At present, AFOP has no unified diagnostic criteria, the age of onset ranged from 50 to 60 years old, some were children (4). The most common symptoms of AFOP were fever, cough, and dyspnea. Bilateral consolidations and GGO usually can be seen on chest CT images (5). In addition, its original chest CT finding could be a solitary nodule with an air bronchogram, and change to diffuse lung opacities over time (6). So, symptoms and imaging manifestations of AFOP were unspecific, the pathological diagnosis plays a crucial role. The pathological pattern is characterized by intra-alveolar deposition of fibrin (fibrin ball) and organizing pneumonia in a scattered distribution, and hyaline membrane is absent in the alveoli which can be 
found in DAD. Compared to OP, the alveolar fillers of OP are Masson bodies. Unlike EP, eosinophilic cells in AFOP tissue and peripheral blood are rarely seen (7). But even so, such typical pathological pattern could also be seen in other diseases such as abscesses, tuberculosis, and granulomas (1). Sometimes, the above pathological manifestations were an unspecific reaction responding to a major lesion, because small biopsies could miss the major lesion. However, large biopsies cannot be acquired as convenient as small biopsies, because surgical lung biopsy (SLB) is more expensive and riskier (8). In previous reports, specimens could be obtained in AFOP patients through SLB, transbronchial lung biopsy (TBLB) and PCNB (9). Recently, Shintani et al. (9) reported an AFOP case diagnosed by transbronchial lung cryobiopsy (TBLC). TBLC is a new diagnosis tool in interstitial lung disease, which can get larger and high-quality samples than TBLB and seems to lower complications and mortality rate than SLB (10). TBLC is a new method to get sample for the diagnosis of diffuse parenchymal lung diseases (11) and the diagnostic rate is higher than traditional forceps biopsy $(50.6-100 \%$ vs. $25-65 \%)$ while mortality in 30 days was similar $(<2 \%$ vs. $0 \%)$. No studies compared diagnostic rate of TBLC with SLB, only a systematic review showed approximately $80 \%$ patients undergoing TBLC were suspected of having an interstitial lung disease (12). SLB has higher inhospital mortality than TBLC, which was $1.7 \%$ in elective lung biopsy and $16 \%$ in non-elective and emergency situation (10). In this case, the patient underwent TBLB and PCNB and was diagnosed with AFOP, but the evidence were still insufficient due to small sample, so long-time follow-up is needed. We can attempt to apply TBLC to some patients who are suspected of AFOP in the future.

At present, the treatment protocol for AFOP has not reached a consensus. Glucocorticoids, immunosuppressants, stem cell transplantation, lung transplantation all have been used (4), glucocorticoid was most commonly used. Akhtar et al. (13) proposed a scheme that uses $60 \mathrm{mg}$ of methylprednisolone per day for 5 days, then reduced the dosage to $40 \mathrm{mg}$ per day for 3 months, and then gradually tapered as appropriate. Glucocorticoid shock therapy was also accepted and applied by clinicians $(14,15)$. When the therapeutic effect of glucocorticoid was poor, immunosuppressors can be added into the treatment plan, commonly used immunosuppressors included cyclophosphamide, mycophenolate mofetil, cyclosporine, etc. (16). This patient was treated with glucocorticoids during her first hospitalization but same symptoms reoccurred when the dosage of glucocorticoid was tapered. We considered progressive disease and the situation was associated with untreated MDS or AML, so glucocorticoid shock therapy and immunosuppressors were administered to control the lung lesions more quickly and further treatment of hematopathy. After the above treatments, her symptoms were improved obviously. CAG chemotherapy was initiated, but PJP appeared after chemotherapy. It can be speculated to be associated with a significant decrease in leukocyte and lymphocyte count after chemotherapy, and her situation was improved after using cotrimoxazole. So, patients who suffered from AFOP with MDS or AML should pay attention to the relationship between the two diseases. When routine treatment was ineffective, controlling the activity of MDS or AML may improve the condition. Some hypotheses speculate that the mechanisms include production of neutrophil superoxide anion and immune abnormalities, and some patients combined with chromosomal abnormalities such as $46 \mathrm{XY},+1, \mathrm{~d}(1 ; 7)$ (q10:p10) which may contribute to this situation (17). But in this case no chromosomal abnormalities or immune abnormalities were found.

The prognosis of AFOP was poor. Beasley et al. (1) found that the overall mortality was $>50 \%$ and patients who received mechanical ventilation all died. Gomes et al. (18) found the overall mortality was $53.8 \%$ while $23.1 \%$ of patients with invasive mechanical ventilation died in overall populations. The median survival time was 78 months. Our patient survives at 25 months' follow up since suffering from the disease and is still alive with treatment, we will continue to pay close attention to her situation.

\section{Conclusions}

In general, we report a case of AFOP with AML who developed PJP after chemotherapy. Combination of Glucocorticoid, immunosuppressor, chemotherapy, and antibiotics was effective. But it is still unclear whether patients with blood disease would affect their progress and prognosis, and the treatment plan has not yet reached a consensus. Therefore, more relevant research should be carried out in the future.

\section{Acknowledgments}

Funding: None. 


\section{Footnote}

Reporting Checklist: The authors have completed the CARE reporting checklist. Available at http://dx.doi.org/10.21037/ apm-20-2344

Conflicts of Interest: All authors have completed the ICMJE uniform disclosure form (available at http://dx.doi. org/10.21037/apm-20-2344). The authors have no conflicts of interest to declare

Ethical Statement: The authors are accountable for all aspects of the work in ensuring that questions related to the accuracy or integrity of any part of the work are appropriately investigated and resolved. All procedures performed in studies involving human participants were in accordance with the ethical standards of the institutional and national research committees, and with the Helsinki Declaration (as revised in 2013). Informed consent was obtained from the patient for the publication.

Open Access Statement: This is an Open Access article distributed in accordance with the Creative Commons Attribution-NonCommercial-NoDerivs 4.0 International License (CC BY-NC-ND 4.0), which permits the noncommercial replication and distribution of the article with the strict proviso that no changes or edits are made and the original work is properly cited (including links to both the formal publication through the relevant DOI and the license). See: https://creativecommons.org/licenses/by-nc-nd/4.0/.

\section{References}

1. Beasley MB, Franks TJ, Galvin JR, et al. Acute fibrinous and organizing pneumonia: a histological pattern of lung injury and possible variant of diffuse alveolar damage. Arch Pathol Lab Med 2002;126:1064-70.

2. Nguyen LP, Ahdoot S, Sriratanaviriyakul N, et al. Acute Fibrinous and Organizing Pneumonia Associated With Allogenic Hematopoietic Stem Cell Transplant Successfully Treated With Corticosteroids: A TwoPatient Case Series. J Investig Med High Impact Case Rep 2016;4:2324709616643990.

3. Merrill AL, Smith H. Myelodysplastic Syndrome and Autoimmunity: A Case Report of an Unusual Presentation of Myelodysplastic Syndrome. Case Rep Hematol 2011;2011:560106.

4. Kuza C, Matheos T, Kathman D, et al. Life after acute fibrinous and organizing pneumonia: a case report of a patient 30 months after diagnosis and review of the literature. J Crit Care 2016;31:255-61.

5. Garcia BA, Goede T, Mohammed T. Acute Fibrinous Organizing Pneumonia: A Case Report and Literature Review. Curr Probl Diagn Radiol 2015;44:469-71.

6. Kobayashi H, Sugimoto C, Kanoh S, et al. Acute fibrinous and organizing pneumonia: initial presentation as a solitary nodule. J Thorac Imaging 2005;20:291-3.

7. Santos C, Oliveira RC, Serra P, et al. Pathophysiology of acute fibrinous and organizing pneumonia - Clinical and morphological spectra. Pathophysiology 2019;26:213-7.

8. Feng AN, Cai HR, Zhou Q, et al. Diagnostic problems related to acute fibrinous and organizing pneumonia: misdiagnosis in 2 cases of lung consolidation and occupying lesions. Int J Clin Exp Pathol 2014;7:4493-7.

9. Shintani R, Oda T, Niwa T, et al. Transbronchial lung cryobiopsy in idiopathic acute fibrinous and organizing pneumonia. Respir Med Case Rep 2019;28:100888.

10. Ravaglia C, Bonifazi M, Wells AU, et al. Safety and Diagnostic Yield of Transbronchial Lung Cryobiopsy in Diffuse Parenchymal Lung Diseases: A Comparative Study versus Video-Assisted Thoracoscopic Lung Biopsy and a Systematic Review of the Literature. Respiration 2016;91:215-27.

11. Hetzel J, Maldonado F, Ravaglia C, et al. Transbronchial Cryobiopsies for the Diagnosis of Diffuse Parenchymal Lung Diseases: Expert Statement from the Cryobiopsy Working Group on Safety and Utility and a Call for Standardization of the Procedure. Respiration 2018;95:188-200.

12. Johannson KA, Marcoux VS, Ronksley PE, et al. Diagnostic Yield and Complications of Transbronchial Lung Cryobiopsy for Interstitial Lung Disease. A Systematic Review and Metaanalysis. Ann Am Thorac Soc 2016;13:1828-38.

13. Akhtar A, Abideen Z. Acute fibrinous and organizing pneumonia masquerading as a lower respiratory tract infection: a case report and review of the literature. BMC Res Notes 2015;8:38.

14. Xu XY, Chen F, Chen C, Sun HM, Zhao BL. Acute fibrinous and organizing pneumonia: A case report and literature review. Exp Ther Med 2016;12:3958-62 .

15. Chan ED, Kalayanamit T, Lynch DA, et al. Mycoplasma pneumoniae-associated bronchiolitis causing severe restrictive lung disease in adults: report of three cases and literature review. Chest 1999;115:1188-94.

16. Al-Khouzaie TH, Dawamneh MF, Hazmi AM. Acute 
fibrinous and organizing pneumonia. Ann Saudi Med 2013;33:301-3.

17. Yamamoto M, Murata K, Kiriu T, et al. Acute Fibrinous and Organizing Pneumonia with Myelodysplastic Syndrome: Corticosteroid Monotherapy Led to Successful
Ventilator Weaning. Intern Med 2016;55:3155-9.

18. Gomes R, Padrao E, Dabo H, et al. Acute fibrinous and organizing pneumonia: A report of 13 cases in a tertiary university hospital. Medicine (Baltimore) 2016;95:e4073.

Cite this article as: Lin M, Zhang Y, Qiu Y. Acute fibrinous and organizing pneumonia with myelodysplastic syndrome and pneumocystis jiroveci pneumonia: a case report. Ann Palliat Med 2021;10(7):8396-8402. doi: 10.21037/apm-20-2344 